\title{
Fast Track THR: One Hospital's Experience with a 2-Day Length of Stay Protocol for Total Hip Replacement
}

\author{
Lawrence V. Gulotta, MD • Douglas E. Padgett, MD • Thomas P. Sculco, MD • Michael Urban, MD, PhD • \\ Stephen Lyman, PhD • Bryan J. Nestor, MD
}

Received: 5 January 2011/Accepted: 25 April 2011/Published online: 2 July 2011

(C) The Author(s) 2011. This article is published with open access at Springerlink.com

\begin{abstract}
Background Current trends in total joint replacement have focused on shorter hospital stays. Purpose This study aimed to determine if a pathway for total hip replacement (THR) with the goal of a 2-day discharge (fast track) is safe and effective compared to our traditional pathway (control). Methods One hundred forty-nine patients undergoing unilateral, uncomplicated, THR were enrolled in an accelerated postoperative pathway and 134 were enrolled in the traditional pathway.
\end{abstract}

Each author certifies that he or she has no commercial associations (e.g., consultancies, stock ownership, equity interest, patent/licensing arrangements, etc.) that might pose a conflict of interest in connection with the submitted article.

Each author certifies that his or her institution has approved the human protocol for this investigation and that all investigations were conducted in conformity with ethical principles of research. This study was approved by the Institutional Review Board at the Hospital for Special Surgery. Investigation performed at the Hospital for Special Surgery.

Level of Evidence: Level II: Prospective Cohort Study

L. V. Gulotta, MD

Sports Medicine and Shoulder Service,

Hospital for Special Surgery, New York, NY, USA

D. E. Padgett, MD.T. P. Sculco, MD.B. J. Nestor, MD ( $ه)$

Arthroplasty Service,

Hospital for Special Surgery,

535 E 70th Street,

New York, NY 10021, USA

e-mail: nestorb@hss.edu

M. Urban, MD, $\mathrm{PhD}$

Department of Anesthesiology,

Hospital for Special Surgery, New York, NY, USA

S. Lyman, $\mathrm{PhD}$

Department of Epidemiology and Biostatistics,

Hospital for Special Surgery, New York, NY, USA
Patients were followed prospectively and outcomes included hospital length of stay, intra- and postoperative complications, readmissions, reoperations. A statistical model was created to determine factors predictive of a 2-day discharge. Results At 1 year, there were no differences in complications, readmissions, or reoperations. The average length of stay decreased from 4.1 to 2.6 days $(p<0.0001)$. In the fast track group, $58 \%$ of patients were discharged home within 2 days. Barriers to a 2-day discharge were postoperative pain, nausea, and dizziness. The only preoperative factor that was predictive of a 2-day discharge was hypertension. Conclusions In a select group of patients, a protocol that allows for a 2-day discharge following THR is safe and effective.

Keywords total hip replacement - postoperative protocol accelerated discharge $\cdot$ clinical pathway

\section{Introduction}

Current trends in total joint replacement, specifically total hip replacement (THR), have focused on less-invasive surgical approaches with the intent of providing quicker rehabilitation and shorter hospital stays [2]. The extent to which these innovative surgical approaches have influenced these goals remains unclear. Regardless, public attention has focused on these objectives and, increasingly, patients are requesting these procedures with the goal of a shorter hospital stay in mind [19, 21]. Additionally, the economic realities of providing medical care have motivated hospitals to reduce their average length of stay for routine procedures [14].

In the USA, the average hospital length of stay following THR has decreased from several weeks, to between 3 and 6 days depending on the region of the country and medical center involved [1,21]. While this decrease is in part due to the expanding role of rehabilitation centers in postoperative care, studies have found that clinical pathways can also help reduce hospital stays and improve the chances of patients being discharged home $[2$, 
$11,16,18,20]$. These pathways are effective because they align the goals of the patient and caregiver, and outline a standardized plan to achieve these goals.

Clinical pathways have been become prevalent in joint replacement surgery $[6,11,13,16-18,20]$. Kim et al. performed a meta-analysis on 11 studies that evaluated the effectiveness of clinical pathways for total knee and total hip replacements [11]. They found that implementing a clinical pathway significantly reduced the average length of stay. They also found that clinical pathways either reduced the incidence of complications or, at least, did not increase these events. While reduced hospital stays meet the shortterm goals of early rehabilitation and limiting hospital costs, there is concern that discharging patients home too early can result in unrecognized perioperative complications that ultimately affect patient's long-term outcome.

An existing clinical pathway for THR at our hospital was modified with the goal of reducing hospital stays to 2 days in a safe and effective manner. We refer to this pathway as the "Fast Track". The purpose of this study was threefold. First, we aimed to determine if patients enrolled in the fast track pathway had a shorter length of stay when compared to matched controls who experienced the standard postoperative pathway. Second, we aimed to report on the safety and feasibility of this modified clinical pathway with respect to perioperative complications and complaints. And third, we aimed to determine what barriers exist to a 2day discharge, and to determine if any predictors for success could be identified. The hypothesis for this prospective, nonrandomized clinical study is that a select group of patients undergoing THR can be discharged from the hospital 2 days after surgery, without any increase in complications or adverse effects on short-term clinical outcomes.

\section{Methods}

Between 2004 and 2006, 149 patients undergoing unilateral, uncomplicated, THR by one of the three senior arthroplasty surgeons were enrolled in this prospective study to examine the safety and efficacy of a 2-day length of stay following THR. These patients made up the fast track cohort. During the same time period, 134 patients undergoing THR by another senior arthroplasty surgeon were matched for age, sex, body mass index (BMI), and comorbidities. This control group of patients followed our hospital's traditional clinical pathway following THR. The traditional pathway was based on a discharge goal of 4 days. The study was approved by the Institutional Review Board at our hospital and all patients consented to participate.

Patients were selected for enrollment in the study based on defined inclusion criteria as follows: age between 18 and 70 years undergoing uncomplicated, unilateral, primary total hip replacement surgery; American Society of Anesthesiologists (ASA) Class II or less with no significant comorbidities and no history of myocardial infarction, pulmonary embolism, or deep venous thrombosis; BMI less than 35; preoperative hemoglobin greater than $13.0 \mathrm{~g} /$ $\mathrm{dl}$; preoperative ambulation without a cane, or over $1,500 \mathrm{ft}$ with a cane; excellent social support as determined by a social worker; and most importantly, patients needed to be motivated to comply with the study protocol.

Exclusion criteria for the study included patients with inflammatory arthritis, complex THR, and medical comorbidities such as a history of a myocardial infarction, pulmonary embolus, or deep venous thrombosis (DVT) such that they were not candidates for multi-modal DVT prophylaxis with aspirin and intermittent compression boots. Patients were also excluded if they did not have someone at home that could stay with them after they were discharged home, or if they had no desire to participate in the pathway. The inclusion and exclusion criteria are outlined in Table 1. This set of inclusion and exclusion criteria applied to patients enrolled in either cohort, fast track or control. All patients in both the fast track group, and the control group were discharged home and not to a rehabilitation facility.

A multidisciplinary team including representatives from nursing, physical therapy, social services, anesthesiology, hospital administration, and orthopedic surgery were involved in development of the fast track protocol. The goal was to develop a clinical pathway aimed at a 2-day length of stay without compromising the standard of care at our hospital. Appropriate daily patient goals were outlined and postoperative orders were modified and standardized to meet these goals. The new protocol, fast track, was devised from a pre-existing clinical pathway with a number of modifications (Table 2). Ambulation with physical therapy began within $6 \mathrm{~h}$ of surgery in the fast track pathway rather

Table 1 Inclusion and exclusion criteria for participation in the fast track program

Inclusion criteria

Age 18-70 years old

Unilateral, primary THR

ASA class II or less

$\mathrm{BMI}<32$

Preoperative hemoglobin $>13 \mathrm{~g} / \mathrm{dl}$

Excellent social support

Preoperative ambulation without cane, or $>1,500 \mathrm{ft}$ with cane.

Motivated to comply with fast track protocol
Exclusion criteria

Inflammatory Arthritis

DDH with high hip center, or concomitant procedure such as removal of hardware at time of THR

History of myocardial infarction, pulmonary embolus, deep venous thrombosis, diabetes mellitus

Unable to arrange to have someone stay with patient after discharge 
Table 2 Patient goals by postoperative day

\begin{tabular}{lll}
\hline Day of surgery & Postoperative day 1 & Postoperative day 2 \\
\hline $\begin{array}{l}\text { First or second case of day } \\
\text { Ambulate with PT, progress to WBAT } \\
\text { with walker as tolerated }\end{array}$ & $\begin{array}{l}\text { Discontinue surgical drain and Foley catheter } \\
\text { PT in morning, attempt to progress to crutches } \\
\text { or cane }\end{array}$ & $\begin{array}{l}\text { Surgical dressing removed } \\
\text { PT in morning, progress to cane and work } \\
\text { on stairs/transfers }\end{array}$ \\
Discont for pain control & Discontinue epidural PCA at 10 am & $\begin{array}{l}\text { If cleared by medical, surgical, and } \\
\text { PT teams } \rightarrow \text { discharged home }\end{array}$ \\
Light liquids for lunch & PT in the afternoon with similar goals, progress & $\begin{array}{l}\text { Study coordinator calls home the day after } \\
\text { discharge to screen for care issues }\end{array}$
\end{tabular}

Begin ECASA for DVT prophylaxis

$P T$ physical therapy, PCA patient-controlled analgesic, ECASA enteric-coated aspirin, DVT deep venous thrombosis, WBAT weight bear as tolerated

than beginning the day after surgery as was the protocol in the traditional pathway. Patients ambulated two additional times on the day of surgery. To facilitate physical therapy on the day of surgery, a fast track patient's surgery was scheduled for either the first or second case of the day. Subsequently, patients were seen by physical therapy twice each day until the time of discharge. Acute postoperative pain management was systemized such that each patient received a dilaudid and marcaine epidural patient-controlled analgesia (PCA) that was automatically discontinued on the morning of postoperative day 1 if the patient's pain visual analog score (VAS) was 2 or less. In the traditional pathway, the epidural PCA was routinely discontinued on postoperative day 2. All patients received enteric-coated aspirin, $325 \mathrm{mg}$ daily, and intermittent compression boots for DVT prophylaxis and were discharged home with arrangements for a physical therapist to work with the patient at home upon discharge. Finally, a study coordinator conducted a phone interview the day after discharge and 1 week after discharge to ensure the patients were doing well and to screen for complications.

Once the pathway was developed, additional patient education material and a modification to an existing preoperative education class were created for patients and their families to inform them about the fast track protocol. Patients were counseled regarding pain control, physical therapy regimens, nausea management, and techniques for preventing atelectasis. Daily goals were outlined to the patients and the strategies for attaining these goals were discussed.

Data was extracted from the Collaborative Orthopedic Replacement Registry including information regarding demographics, length of stay, intra-operative and postoperative complications. Hospital charts were reviewed for readmissions and reoperations, reasons for delayed discharge, daily VAS, narcotic and anti-emetic usage, and the occurrences of dizziness and nausea that either shortened or precluded a patient's participation in physical therapy. Patients were followed for 1 year following their index surgery.

A chi-squared test was used to determine that had no difference between groups based on sex. Likewise, an independent samples $t$ test was used to demonstrate no significant difference between groups based on age and BMI. For testing of hypotheses, inferential statistics were calculated using a Fisher's exact test for risk of readmission and reoperation between the fast track and standard cohorts. An independent samples $t$ test was used to calculate differences between the groups with regard to length of stay. A prediction model was built using multiple logistic regression equation using only the fast track patients. Age, sex, and BMI were forced into this model while all other potential predictors were evaluated first with univariate analyses (Fisher's or t tests as appropriate). Those deemed to be associated $(p<0.25)$ with failure to achieve early discharge were eligible for inclusion. Those that achieved statistical significance $(p<0.05)$ were retained in the model. All analyses were performed using SPSS for Windows version 13.0 (Chicago, IL).

\section{Results}

There were no significant differences between the fast track and control groups in terms of age, gender, BMI, primary diagnosis, comorbidities, or femoral fixation of the implant (Tables 3 and 4).

\section{Length of Stay}

The average length of stay in the fast track group was $2.6 \pm$ 0.9 days, which was significantly shorter than for patients in the control group $(4.1 \pm 1.5$ days; $p<0.0001)$. Of the patients participating in the fast track protocol, $58 \%$ were successfully discharged home within 2 days of surgery, and $73 \%$ were discharged home within 3 days.

Table 3 Results of the matching process

\begin{tabular}{llll}
\hline & $\begin{array}{l}\text { Fast track } \\
(n=149)\end{array}$ & $\begin{array}{l}\text { Matched controls } \\
(n=134)\end{array}$ & $p$ value \\
\hline Age in years & $50.3 \pm 8.9$ & $52.3 \pm 8.5$ & 0.06 \\
Female sex & $34.2 \%$ & $40.3 \%$ & 0.29 \\
Body mass index & $26.9 \pm 5.9$ & $27.2 \pm 5.8$ & 0.65 \\
Primary diagnosis of OA & $85.9 \%$ & $86.6 \%$ & 0.87 \\
Any comorbidities & $49.0 \%$ & $47.0 \%$ & 0.74 \\
Uncemented implant & $91.3 \%$ & $95.5 \%$ & 0.15 \\
\hline
\end{tabular}


Table 4 Fast track cohort compared to matched controls who underwent a standard postoperative care protocol

$\begin{array}{ll}\begin{array}{l}\text { Fast track } \\ (n=149)\end{array} & \begin{array}{l}\text { Matched controls } \\ (n=134)\end{array}\end{array}$

\begin{tabular}{lccc}
\hline Length of stay in days & $2.6 \pm 0.9$ & $4.1 \pm 1.5$ & $<0.0001$ \\
\multicolumn{4}{l}{ Common post-op complaints/complications } \\
\multicolumn{4}{l}{} \\
Reoperation & $2(1.3 \%)$ & $1(0.8 \%)$ & \\
Readmission & $5(3.4 \%)$ & $1(0.8 \%)$ & 0.99 \\
Local complications & $7(4.7 \%)$ & $7(5.2 \%)$ & 0.22 \\
Dislocation & $3(2.0 \%)$ & $2(1.5 \%)$ & 0.84 \\
Drainage & $1(0.7 \%)$ & $2(1.5 \%)$ & 0.99 \\
Gout & None & $2(1.5 \%)$ & 0.22 \\
Respiratory dysfunction & $1(0.7 \%)$ & None & 0.99 \\
Pulmonary embolus & $1(0.7 \%)$ & None & 0.99 \\
Atrial fibrillation & $1(0.7 \%)$ & $1(0.8 \%)$ & 0.99 \\
Urinary tract infection & $1(0.7 \%)$ & $1(0.8 \%)$ & 0.99 \\
Dizziness & $28(18.8 \%)$ & $56(41.8 \%)$ & $<0.0001$ \\
Nausea & $20(13.4 \%)$ & $29(21.6 \%)$ & 0.07 \\
& & &
\end{tabular}

Postoperative pain (VAS):

$\begin{array}{llll}\text { Day 0 } & 3.0 \pm 1.9 & 2.7 \pm 1.3 & 0.19 \\ \text { Day 1 } & 2.2 \pm 1.3 & 2.2 \pm 1.1 & 0.90 \\ \text { Day 2 } & 1.8 \pm 1.3 & 1.8 \pm 0.8 & 0.72\end{array}$

Narcotic use:

\begin{tabular}{lrrc} 
Day 0 & $10.1 \%$ & $3.0 \%$ & 0.01 \\
Day 1 & $68.5 \%$ & $32.1 \%$ & $<0.0001$ \\
Day 2 & $66.4 \%$ & $42.5 \%$ & $<0.0001$ \\
Anti-emetic use: & & & \\
Day 0 & $58.1 \%$ & $49.3 \%$ & 0.17 \\
Day 1 & $13.4 \%$ & $18.7 \%$ & 0.24 \\
Day 2 & $4.8 \%$ & $9.0 \%$ & 0.19 \\
\hline
\end{tabular}

Readmissions, Complications, and Complaints

There were five readmissions in the fast track group (3.4\%). Two patients had a dislocation that required closed reduction in the Emergency Department. One patient required femoral revision for a periprosthetic femoral fracture. Another patient underwent acetabular cup revision for early aseptic loosening. One patient was called back to the hospital for a blood transfusion the same day of discharge for significant anemia. That patient was subsequently discharged home later that evening following the transfusion. All five of these patients were discharged home within 2 days of surgery and were therefore classified as successful 2-day discharge patients. There was only one readmission in the control group $(0.8 \%, p=0.22)$. This was for a dislocation, which required open reduction and revision of the prosthesis. There were no differences in the rates of overall complications between the fast track and control groups (Table 4).

There were two major postoperative medical complications in the fast track group. One patient experienced a gastrointestinal bleed on postoperative day 1 requiring transfer to the medical intensive care unit. Another patient had a pulmonary embolus resulting in atrial fibrillation, also on postoperative day 1 . Both patients recovered with medical management and were discharged home within 6 days.
When postoperative complaints were assessed, there was a lower rate of postoperative dizziness in the fast track group (18.8\%) compared to the control group $(41.8 \%, p<$ $0.0001)$. There were higher rates of oral narcotic use in the fast track group on POD\#1 $(68.5 \%$ vs. $32.1 \%, p<0.0001)$ and on POD\#2 $(66.4 \%$ vs. $42.5 \%, p<0.0001)$ than in the control group.

In $14 \%$ of all study patients, the patients were cleared for discharge home within 2 days of surgery by the medical team and physical therapy; however, arrangements for discharge were not able to be made. A protocol violation occurred for three patients that were originally in the fast track cohort $(2 \%)$ when they were not seen by physical therapy within $6 \mathrm{~h}$ of surgery.

\section{Predictors of a Successful 2-Day Discharge}

When a predictive model was constructed to determine the likelihood of success for a 2-day discharge for patients enrolled in the fast track pathway, the only preoperative predictor that could be determined was the presence of hypertension. Patients with no history of hypertension were 3.25 times more likely to be discharged home within 2 days compared to those with hypertension (95\% CI, 1.39-7.60; $p<0.006$; Table 5).

Patients who experienced no postoperative nausea were 3.41 times more likely to be discharged within 2 days than those who did (95\% CI, $1.3-8.92 ; p=0.01)$. Patients who did not experience dizziness were 16.1 times more likely to be successful than those who did not (95\% CI, 5.58-46.6; $p<0.0001)$.

\section{Discussion}

There were no differences in the complication, readmission, and reoperation rates for the fast track group compared to the control group in this study. We feel this proves that a 2-day discharge following uncomplicated THR in a select group of relatively healthy patients is safe. Patients enrolled in the fast track protocol had an average length of stay of 2.6 days, which was significantly shorter than an average length of stay of 4.1 days for the matched control group.

Table 5 Predictive model for successful discharge at 2 days for patients participating in the fast track pathway

\begin{tabular}{lccc}
\hline Variable & Odds ratio & $95 \%$ CI & $p$ value \\
\hline Age & 0.99 & $0.96,1.03$ & 0.71 \\
Male sex & 1.81 & $0.93,3.52$ & 0.08 \\
BMI & 1.06 & $0.99,1.13$ & 0.06 \\
No hypertension & 3.25 & $1.39,7.60$ & 0.006 \\
No nausea & 3.41 & $1.30,8.92$ & 0.01 \\
No dizziness & 16.1 & $5.58,46.6$ & $<0.0001$ \\
$\begin{array}{l}\text { Narcotics on } \\
\text { day 1 }\end{array}$ & 3.43 & $1.05,11.2$ & 0.04 \\
$\begin{array}{l}\text { Narcotics on } \\
\text { day 2 }\end{array}$ & 1.80 & $1.00,3.24$ & 0.05 \\
\hline
\end{tabular}


This suggests that the program is effective in reducing length of stay. The most common barriers to a successful 2day discharge were postoperative nausea and dizziness that interfered with physical therapy.

While the overall readmission rate was not statistically different between the fast track and control groups, all five patients that were readmitted in the fast track group were successfully discharged from the hospital within 2 days of their surgery. No fast track patients that were discharged after 2 days were readmitted. One patient was readmitted for a blood transfusion that may have been prevented had the patient not been discharged within 2 days. However, our protocol had an infrastructure in place to follow-up and identify patients that require further medical care following discharge and the transfusion was addressed in a timely manner. Of the other four patients who were readmitted, there were two dislocations and one periprosthetic fracture. The relationship that these complications have with a shortened hospital stay is uncertain. Previous authors have suggested that a shortened hospital stay is associated with a higher dislocation rate; however, this did not reach significance in the present study [13].

Several factors are involved in developing a successful clinical pathway following total joint procedures. Probably the most important factor in our study was patient selection criteria. Jain et al. found that hypertension, diabetes, and obesity were each independent risk factors for postoperative complications [10]. Forrest et al. found that only patient age was correlated to length of stay, but age and diabetes were predictive of discharge to a rehabilitation center [8]. Bozic et al. also found older age and higher ASA Class were predictive of a discharge to a rehabilitation facility [5]. Hayes et al. found that patient's age, female gender, and number of comorbidities influenced length of stay [9].

The findings in our study are similar to those previously reported. While patients with comorbidities such as diabetes, previous myocardial infarction, and morbid obesity, and patients with an ASA of Class II or higher were excluded from participation, we did find that patients with hypertension were over three times less likely to be discharged home within 2 days when enrolled in the fast track protocol. We currently do not include hypertension as a contraindication for participation in the fast track protocol.

While many studies have shown that clinical pathway can safely reduce stays to within 3-5 days of surgery, very few have looked at earlier discharges. Berger et al. showed that 97 of 100 patients who underwent two-incision minimally invasive THR were discharged home on the day of surgery [4]. They reported no dislocations, reoperations, or readmissions to the hospital. More recently in a follow-up to that study, the same group reported $9 \%$ rate of either readmission or emergency room visits following discharge [3]. However, in those studies, it is unclear if all patients were discharged home as was the case in the current study. In our study, there were two major medical complications in the fast track group in addition to the previously mentioned five readmissions. One patient had a gastrointestinal bleeding and another had a pulmonary embolism resulting in atrial fibrillation. Both complications occurred on postoperative day 1 and were managed with little subsequent morbidity.

Perioperative anesthesia and its complications are other factors that influence length of stay. Postoperative pain, nausea, and dizziness that interfered with physical therapy were the main reasons for an unsuccessful 2-day discharge. In our study, all patients were placed on an epidural PCA. Continuous epidurals can control pain in the immediate postoperative period, and can allow earlier attainment of functional goals when compared to general anesthesia [2]. The PCA was routinely discontinued after $24 \mathrm{~h}$ and the patients were switched to oral pain medication by the end of postoperative day 1 in the fast track group. It is possible that patients with an unsuccessful 2-day discharge were more sensitive to the medications in the epidural, thus resulting in increased rates of nausea and dizziness and prolonged stays. Also, beginning ambulation with physical therapy within $6 \mathrm{~h}$ of surgery may contribute to nausea and dizziness as well. These findings raise the question as to whether more aggressive, preemptive anti-emetic usage is warranted in patients eligible for fast track discharge. Future research will explore the use of a low opioid pathway to eliminate these complications.

Another factor in a successful clinical pathway is preoperative education. McGregor et al. found that preoperative classes and booklets reduced hospital stays by 3 days, and resulted in higher patient satisfaction due to more realistic expectations of surgery [15]. Daltroy et al. also showed that patients undergoing preoperative education, including psychosocial preparation, had reduced hospital stays and required less pain medication postoperatively [7]. The fast track pathway presented in this study includes a mandatory preoperative education class that is taught by a multidisciplinary team [12].

We acknowledge several limitations in our study. The patients were not randomized and that may have contributed to a patient selection bias. Second, actual cost savings were not calculated for this study. It is possible that the added costs of physical therapy, nursing, and social work needed to institute the fast track pathway did not outweigh the savings that early discharges afforded. Third, this study was conducted at a large orthopedic specialty hospital that performs over approximately 2,500 THR's annually. This represents a small subset of that experience and the results may not be generalizable to hospitals with smaller volume and a less specialized staff. And finally, there is the possibility that significant differences in complications and readmissions were not found in this study due to its sample size limitations.

Our findings support our hypothesis that a 2-day length of stay protocol following THR can be safe and effective. The pathway reduced the overall length of stay in our hospital regardless of whether patients successfully met the 2-day discharge goal or not. The complications following surgery were comparable to those previously reported for patients undergoing THR. Further research is needed to determine ways to reduce the incidence of postoperative nausea and dizziness, since these were the most important factors that delayed discharge. 
Open Access This article is distributed under the terms of the Creative Commons Attribution Noncommercial License which permits any noncommercial use, distribution, and reproduction in any medium, provided the original author(s) and source are credited.

\section{References}

1. Antoniou J, Martineau PA, Filion KB, et al. In-hospital cost of total hip arthroplasty in Canada and the United States. $J$ Bone Joint Surg Am. 2004; 86-A(11):2435-2439.

2. Berend KR, Lombardi AV,Jr, Mallory TH. Rapid recovery protocol for peri-operative care of total hip and total knee arthroplasty patients. Surg Technol Int. 2004;13:239-247.

3. Berger RA, Kusuma SK, Sanders SA, Thill ES, Sporer SM. The feasibility and perioperative complications of outpatient knee arthroplasty. Clin Orthop Relat Res. 2009;467(6):1443-1449.

4. Berger RA. Total hip arthroplasty using the minimally invasive twoincision approach. Clin Orthop Relat Res. 2003;(417): 232-241.

5. Bozic KJ, Wagie A, Naessens JM, Berry DJ, Rubash HE. Predictors of discharge to an inpatient extended care facility after total hip or knee arthroplasty. J Arthroplasty. 2006;21(6 Suppl 2):151-156.

6. Brunenberg DE, van Steyn MJ, Sluimer JC, Bekebrede LL, Bulstra SK, Joore MA. Joint recovery programme versus usual care: an economic evaluation of a clinical pathway for joint replacement surgery. Med Care. 2005;43(10):1018-1026.

7. Daltroy LH, Morlino CI, Eaton HM, Poss R, Liang MH. Preoperative education for total hip and knee replacement patients. Arthritis Care Res. 1998;11(6):469-478.

8. Forrest G, Fuchs M, Gutierrez A, Girardy J. Factors affecting length of stay and need for rehabilitation after hip and knee arthroplasty. J Arthroplasty. 1998;13(2):186-190.

9. Hayes JH, Cleary R, Gillespie WJ, Pinder IM, Sher JL. Are clinical and patient assessed outcomes affected by reducing length of hospital stay for total hip arthroplasty? J Arthroplasty. 2000;15 (4):448-452.

10. Jain NB, Guller U, Pietrobon R, Bond TK, Higgins LD. Comorbidities increase complication rates in patients having arthroplasty. Clin Orthop Relat Res. 2005;(435):232-238.
11. Kim S, Losina E, Solomon DH, Wright J, Katz JN. Effectiveness of clinical pathways for total knee and total hip arthroplasty: literature review. J Arthroplasty. 2003;18(1):69-74.

12. Mancuso CA, Graziano S, Briskie LM, et al. Randomized trials to modify patients' preoperative expectations of hip and knee arthroplasties. Clin Orthop Relat Res. 2008;466 (2):424-431.

13. Mauerhan DR, Lonergan RP, Mokris JG, Kiebzak GM. Relationship between length of stay and dislocation rate after total hip arthroplasty. J Arthroplasty. 2003;18(8):963-967.

14. Mayes R, Hurley RE. Pursuing cost containment in a pluralistic payer environment: from the aftermath of Clinton's failure at health care reform to the Balanced Budget Act of 1997. Health Econ Policy Law. 2006;1(Pt 3):237-261.

15. McGregor AH, Rylands H, Owen A, Dore CJ, Hughes SP. Does preoperative hip rehabilitation advice improve recovery and patient satisfaction? J Arthroplasty. 2004;19(4):464-468.

16. Peterson MG, Cioppa-Mosca J, Finerty E, Graziano S, King S, Sculco TP. Effectiveness of best practice implementation in reducing hip arthroplasty length of stay. J Arthroplasty. 2008;23 (1):69-73.

17. Rissanen P, Aro S, Paavolainen P. Hospital- and patient-related characteristics determining length of hospital stay for hip and knee replacements. Int J Technol Assess Health Care. 1996;12 (2):325-335.

18. Siggeirsdottir K, Olafsson O, Jonsson H, Iwarsson S, Gudnason V, Jonsson BY. Short hospital stay augmented with education and home-based rehabilitation improves function and quality of life after hip replacement: randomized study of 50 patients with 6 months of follow-up. Acta Orthop. 2005;76(4):555562.

19. Vail TP, Callaghan JJ. Minimal incision total hip arthroplasty. $J$ Am Acad Orthop Surg. 2007;15(12):707-715.

20. Walter FL, Bass N, Bock G, Markel DC. Success of clinical pathways for total joint arthroplasty in a community hospital. Clin Orthop Relat Res. 2007;457:133-137.

21. Wright JM, Crockett HC, Delgado S, Lyman S, Madsen M, Sculco TP. Mini-incision for total hip arthroplasty: a prospective, controlled investigation with 5-year follow-up evaluation. $J$ Arthroplasty. 2004;19(5):538-545. 